\title{
Motion of a hot particle in viscous fluids
}

\author{
Naomi Oppenheimer \\ Department of Mechanical and Aerospace Engineering, Princeton University, Princeton, \\ New Jersey 08544, USA \\ Shahin Navardi* \\ Department of Mechanical Engineering, Texas Tech University, Lubbock, Texas 79409, USA
}

\author{
Howard A. Stone ${ }^{\dagger}$ \\ Department of Mechanical and Aerospace Engineering, Princeton University, Princeton, \\ New Jersey 08544, USA \\ (Received 23 January 2016; published 18 May 2016)
}

\begin{abstract}
We study the motion of a hot particle in a viscous liquid at low Reynolds numbers, which is inspired by recent experiments with Brownian particles heated by a laser. The difference in temperature between a particle and the ambient fluid causes a spatial variation of the viscosity in the vicinity of the solid body. We derive a general analytical expression determining the force and the torque on a particle for low Péclet numbers by exploiting the Lorentz reciprocal theorem. For small temperature and viscosity variations, a perturbation analysis is implemented to evaluate the leading-order correction to the hydrodynamic force and torque on the particle. The results are applied to describe dynamics of a uniformly hot spherical particle and to spherical particles with a nonuniform surface temperature described by dipole and quadrupole moments. Among other results, we find for dipolar thermal fields that there is coupling of the translational and rotational motions when there are local viscosity variations; such coupling is absent in an isothermal fluid.
\end{abstract}

DOI: 10.1103/PhysRevFluids.1.014001

\section{INTRODUCTION}

There are many problems of colloid science and physicochemical hydrodynamics where temperature gradients are relevant to the motion of suspended particles; see, e.g., Refs. [1-4]. The most common case is probably thermophoresis where rigid particles move in response to an imposed temperature gradient; the underlying cause for the relative motion of particles and fluid is typically electrical effects that are tied to the (thermally dependent) diffusion of the cations and anions in solution past the charged surface of a particle. The standard analyses of these problems simply assume the viscosity of the solution is constant; see, e.g., Ref. [5]. More recently, the Brownian motion of a small heated sphere, a so-called hot particle, has been investigated [6,7]. The authors discovered that the particle diffusion has distinct features from the case of diffusion in isothermal systems. In the hot particle dynamics the spatially varying temperature field produces variations in the rheological properties of the medium around the particle, which modifies the diffusion coefficient of the particle. This problem motivates the theoretical study reported here. We present an asymptotic calculation for the corrections to the hydrodynamic force and torque on a particle as a consequence of a variation of viscosity with temperature in the neighborhood of the particle.

\footnotetext{
"Current address: Department of Mechanical Engineering, Cleveland State University, Cleveland, Ohio 44115, USA.

${ }^{\dagger}$ Corresponding author: hastone@ princeton.edu
} 
Such results are one element of a complete theory of Brownian motion in systems with temperature gradients.

The effect of viscosity gradients due to temperature has been studied by investigating the motion of individual particles in a liquid in direct response to a local, laser-induced temperature gradient [8]. To model experiments on the motion of a heated particle [7], the continuous spatial variation of viscosity was approximated by Chakraborty et al. [6] using a set of spherical shells of monotonically changing but constant viscosity. Since the solution to the constant viscosity problem is straightforward, multiple such solutions can be stitched together. These studies identify the typical magnitude of the change in the force-velocity relation owing to the change in viscosity.

Thus, although there are approximate formulas for the force on hot particles considering the variation in the viscosity of the surrounding liquid, the available results have been derived using ad hoc approaches. To the best of our knowledge there are no analytical expressions available for the force or torque on a particle that have been derived based on a self-consistent analysis of the equations of low-Reynolds-number hydrodynamics. Formally, a solution to this problem requires a general solution to the Stokes equations considering viscosity as a position-dependent function. Then by applying the boundary conditions for the object, we can evaluate the flow field and eventually the corresponding force and torque on the object. Obviously, numerical solutions can be developed to solve this problem on a case-by-case basis for a given viscosity distribution. We note that the limit of large viscosity differences between the sphere and the ambient, where the variations are confined to a narrow boundary layer near the particle, has also been considered [9].

We seek analytical results by exploiting the Lorentz reciprocal theorem [10, p. 85]. In Sec. II we derive a general analytical expression [Eqs. (18) and (19)] for the hydrodynamic force and torque on an arbitrarily shaped translating and rotating particle for a prescribed, but otherwise arbitrary, viscosity distribution. As written, Eqs. (18) and (19) are too general to be used directly, but they provide a starting point for a perturbation solution. This use of the reciprocal theorem is similar to previous applications to particle motion in non-Newtonian fluids (e.g., Refs. [11-13]), to particle motions incorporating inertial effects in the fluid (e.g., Refs. [12,14]), and to particle motions in electric fields (e.g., Ref. [15]). Thus, in Sec. III we apply a perturbation scheme to the obtained results for small variations of viscosity with temperature, $\eta(T)$, where we introduce the small parameter $\epsilon=-\left.\frac{\Delta T}{\eta_{0}} \frac{d \eta}{d T}\right|_{T_{0}}$ that characterizes the changes of viscosity $\eta$ at reference temperature $T_{0}$ given a typical change in temperature $\Delta T$ between the particle and the fluid far away; the minus sign in $\epsilon$ is introduced since in common liquids $\frac{d \eta}{d T}<0$. Then in Sec. IV we obtain the hydrodynamic force and torque to $O(\epsilon)$ without solving for the detailed flow field at $O(\epsilon)$. Various results are presented based on the thermal characterization of a hot particle in terms of monopole, dipole, and quadrupolar moments. A short summary and discussion are given in Sec. V.

\section{PROBLEM FORMULATION AND SOLUTION}

\section{A. Problem statement}

We present a general theoretical approach to quantify the force on a particle of arbitrary shape that is hotter than its surroundings and produces a local variation of viscosity. We assume that the dynamics are those of a low-Reynolds-number flow. Also, we assume that the advective transport rate of thermal energy is negligible compared to conduction effects, i.e., the Péclet number $\mathrm{Pe}=$ $U a / \kappa \ll 1$, where $U, a$, and $\kappa$ are, respectively, the typical speed and particle size, and $\kappa$ is the thermal diffusivity. As a result, (1) distortions induced by the flow on the temperature field can be neglected, and (2) particle-scale features of the temperature distribution are imprinted on the thermal field in the surrounding fluid. The viscosity in liquids is temperature dependent, and consequently the viscosity varies with position. Therefore, both the flow field and the hydrodynamic force on a translating particle will differ from their respective results in the well-known case of particle motion in a fluid of constant viscosity. 
We denote the position vector as $\boldsymbol{r}$. For an incompressible flow in the limit of low Reynolds numbers, the velocity $\boldsymbol{u}$ and stress $\boldsymbol{\sigma}$ fields are determined by

$$
\boldsymbol{\nabla} \cdot \boldsymbol{u}=0 \quad \text { and } \quad \boldsymbol{\nabla} \cdot \boldsymbol{\sigma}=\mathbf{0} .
$$

For a Newtonian fluid and a (quasisteady state) temperature distribution $T(\boldsymbol{r})$, the stress $\sigma$, pressure $p$, spatially varying viscosity $\eta[T(\boldsymbol{r})]$ or simply $\eta(\boldsymbol{r})$, and the rate of strain tensor $\boldsymbol{E}=\frac{1}{2}\left[\nabla \boldsymbol{u}+(\boldsymbol{\nabla} \boldsymbol{u})^{T}\right]$ are related by

$$
\boldsymbol{\sigma}=-p \boldsymbol{I}+2 \eta(\boldsymbol{r}) \boldsymbol{E}
$$

where $I$ is the identity tensor. Substituting Eq. (2) into Eq. (1) shows that the velocity and pressure fields follow from the solution of

$$
\boldsymbol{\nabla} \cdot \boldsymbol{u}=0 \quad \text { and } \quad-\nabla p+\eta(\boldsymbol{r}) \nabla^{2} \boldsymbol{u}+(\nabla \eta) \cdot\left[\nabla \boldsymbol{u}+(\nabla \boldsymbol{u})^{T}\right]=\mathbf{0} .
$$

In writing these equations we have assumed that temperature-induced density variations, $\Delta \rho$, do not significantly affect the flow; i.e., $\frac{\Delta \rho g a^{2}}{\mu U} \ll 1$, where $a$ is the particle size and $U$ is the typical particle speed.

We are interested in the hydrodynamic force $\boldsymbol{F}$ and torque $\boldsymbol{T}$ on a particle (surface $S_{p}$ ), which are, respectively, given by

$$
\boldsymbol{F}=\int_{S_{p}} \boldsymbol{n} \cdot \boldsymbol{\sigma} d S \quad \text { and } \quad \boldsymbol{T}=\int_{S_{p}} \boldsymbol{r} \wedge(\boldsymbol{n} \cdot \boldsymbol{\sigma}) d S,
$$

where $\boldsymbol{n}$ is the unit normal directed into the fluid.

Our main objective is to quantify the difference of the force and torque on a translating and rotating particle calculated according to Eqs. (1) and (2) to the force and torque on the same shaped particle obtained from the Stokes equations when the viscosity is constant; i.e., $\eta=\eta_{0}$ does not depend on position:

$$
\boldsymbol{\nabla} \cdot \boldsymbol{u}_{0}=0 \quad \text { and } \quad-\nabla p_{0}+\eta_{0} \nabla^{2} \boldsymbol{u}_{0}=\nabla \cdot \boldsymbol{\sigma}_{0}=\mathbf{0}
$$

The subscript 0 refers to quantities calculated for the model problem, i.e., the case of constant viscosity. In this case, for particle translation $\boldsymbol{U}_{0}$ and rotation $\boldsymbol{\Omega}_{0}$, the force and torque are given by $\left(\begin{array}{l}\boldsymbol{F}_{0} \\ \boldsymbol{T}_{0}\end{array}\right)=\boldsymbol{R}_{0} \cdot\left(\begin{array}{l}\boldsymbol{U}_{0} \\ \boldsymbol{\Omega}_{0}\end{array}\right)$, where $\boldsymbol{R}_{0}$ is the resistance tensor (each term is proportional to $\eta_{0}$ ).

\section{B. Remarks about the structure of the solution for a given viscosity distribution}

The solution of Eqs. (5) for the case of constant viscosity is well known and is discussed in many books and research papers. For completeness we make a few remarks about the corresponding structure of the solution of Stokes equations [e.g., Eqs. (3)] when there is a spatial variation of viscosity. Although the ideas and main conclusions mimic those of the constant viscosity case, we are not aware of this characterization having been given previously for the problem at hand.

\section{The form of the velocity field for a translating particle in a fluid with viscosity variations}

To appreciate one feature of the solution to Eqs. (3) for a given viscosity distribution, we consider translation of a particle; i.e., the boundary conditions are $\boldsymbol{u}=\boldsymbol{U}$ on the surface of the particle $S_{p}$ and $\{\boldsymbol{u}, p\} \rightarrow\{\mathbf{0}, 0\}$ as $|\boldsymbol{r}| \rightarrow \infty$. We note that the solution can be expressed as $\boldsymbol{u}(\boldsymbol{r})=\boldsymbol{A}(\boldsymbol{r}) \cdot \boldsymbol{U}$ and $p(\boldsymbol{r})=\boldsymbol{b}(\boldsymbol{r}) \cdot \boldsymbol{U}$, where, since $\boldsymbol{U}$ is an arbitrarily selected velocity vector, the second-order tensor $\boldsymbol{A}(\boldsymbol{r})$ and vector $\boldsymbol{b}(\boldsymbol{r})$ satisfy equations and boundary conditions that are independent of $\boldsymbol{U}$, e.g.,

$$
\boldsymbol{\nabla} \cdot \boldsymbol{A}=0 \quad \text { and } \quad-\nabla \boldsymbol{b}+\eta(\boldsymbol{r}) \nabla^{2} \boldsymbol{A}+(\nabla \eta) \cdot\left[\nabla \boldsymbol{A}+(\boldsymbol{\nabla} \boldsymbol{A})^{T}\right]=\mathbf{0}
$$


These equations are to be solved with $\boldsymbol{A}=\boldsymbol{I}$ on the particle $S_{p}$ and $\{\boldsymbol{A}, \boldsymbol{b}\} \rightarrow\{\mathbf{0 , 0}\}$ as $|\boldsymbol{r}| \rightarrow \infty$. Thus, we see that linearity ensures a solution structure $\boldsymbol{u}(\boldsymbol{r})=\boldsymbol{A}(\boldsymbol{r}) \cdot \boldsymbol{U}$ that has the same qualitative features as for the constant viscosity case.

\section{Symmetry of the resistance tensor for translation}

As a further consequence, we can then conclude that the force-velocity relation for a translating particle will have the form $\boldsymbol{F}=\boldsymbol{R}^{F U} \cdot \boldsymbol{U}$, where $\boldsymbol{R}^{F U}$ is the corresponding resistance tensor. In the case of a constant viscosity fluid it is known for an arbitrarily shaped particle that $\boldsymbol{R}^{F U}$ is symmetric [10]. The same holds true even for an arbitrary viscosity variation as we now show.

Consider an arbitrarily shaped particle and a given viscosity distribution $\eta(\boldsymbol{r})$. Next, consider two different translational motions, which we shall denote $\boldsymbol{U}_{1}$ and $\boldsymbol{U}_{2}$, with the corresponding velocity and stress fields $\left(\boldsymbol{u}_{1}, \boldsymbol{\sigma}_{1}\right)$ and $\left(\boldsymbol{u}_{2}, \boldsymbol{\sigma}_{2}\right)$. We now make our first use of the reciprocal theorem. Note that $\left(\nabla \cdot \sigma_{1}\right) \cdot \boldsymbol{u}_{2}=0$, and so we have $\nabla \cdot\left(\boldsymbol{\sigma}_{1} \cdot \boldsymbol{u}_{2}\right)-\boldsymbol{\sigma}_{1}: \nabla \boldsymbol{u}_{2}=0$; as a result of incompressibility and the symmetry of the stress tensor we obtain

$$
\nabla \cdot\left(\boldsymbol{\sigma}_{1} \cdot \boldsymbol{u}_{2}\right)-2 \eta(\boldsymbol{r}) \boldsymbol{E}_{1}: \boldsymbol{E}_{2}=0 .
$$

Similarly, we can find

$$
\nabla \cdot\left(\boldsymbol{\sigma}_{2} \cdot \boldsymbol{u}_{1}\right)-2 \eta(\boldsymbol{r}) \boldsymbol{E}_{2}: \boldsymbol{E}_{1}=0 .
$$

Subtracting Eq. (8) from Eq. (7) eliminates the viscosity distribution, and we arrive at

$$
\nabla \cdot\left(\sigma_{1} \cdot u_{2}\right)=\nabla \cdot\left(\sigma_{2} \cdot u_{1}\right)
$$

which is the traditional form of the Lorentz Reciprocal Theorem. This intermediate result is identical to the case of a constant viscosity fluid, and so the final steps are also the same. For example, integrating over the fluid domain, using the Divergence Theorem, and applying the boundary conditions lead to

$$
\boldsymbol{F}_{1} \cdot \boldsymbol{U}_{2}=\boldsymbol{F}_{2} \cdot \boldsymbol{U}_{1}
$$

Since $\boldsymbol{F}=\boldsymbol{R}^{F U} \cdot \boldsymbol{U}$, as shown above, then Eq. (10) leads to $\boldsymbol{U}_{2} \cdot \boldsymbol{R}^{F U} \cdot \boldsymbol{U}_{1}=\boldsymbol{U}_{1} \cdot \boldsymbol{R}^{F U} \cdot \boldsymbol{U}_{2}$, from which we conclude that $\boldsymbol{R}^{F U}$ is a symmetric, positive-definite second-order tensor.

Consequently, we should expect the generalized resistance tensor $\boldsymbol{R}$ for the case of an arbitrary viscosity distribution $\eta(\boldsymbol{r})$ to have the same kinds of properties as for the case of constant viscosity. Specifically, similar to the derivation just given, the resistance tensors relating the various combinations of force $\boldsymbol{F}$ and torque $\boldsymbol{T}$ to translational $\boldsymbol{U}$ and angular $\boldsymbol{\Omega}$ velocities, have the properties

$$
\boldsymbol{R}^{F U}=\left(\boldsymbol{R}^{F U}\right)^{T}, \quad \boldsymbol{R}^{T \Omega}=\left(\boldsymbol{R}^{T \Omega}\right)^{T}, \quad \boldsymbol{R}^{F \Omega}=\left(\boldsymbol{R}^{T U}\right)^{T} .
$$

\section{Use of the reciprocal theorem to derive equations for the force and torque}

In order to avoid the complexities of solving the differential Eqs. (1)-(3) arising from the variations of the viscosity with position, we exploit the reciprocal theorem [10]. To relate the solution of the Stokes equations (1) and (5), we begin with $(\nabla \cdot \boldsymbol{\sigma}) \cdot \boldsymbol{u}_{0}=0$ and follow the steps from the previous subsection to arrive at

$$
\nabla \cdot\left(\boldsymbol{\sigma} \cdot \boldsymbol{u}_{0}\right)-2 \eta(\boldsymbol{r}) \boldsymbol{E}: \boldsymbol{E}_{0}=0 .
$$

Similarly $\left(\nabla \cdot \boldsymbol{\sigma}_{0}\right) \cdot \boldsymbol{u}=0$ leads to

$$
\nabla \cdot\left(\boldsymbol{\sigma}_{0} \cdot \boldsymbol{u}\right)-2 \eta_{0} \boldsymbol{E}_{0}: \boldsymbol{E}=0 .
$$

By subtracting Eq. (13) from Eq. (12) we have

$$
\boldsymbol{\nabla} \cdot\left(\boldsymbol{\sigma} \cdot \boldsymbol{u}_{0}\right)-\nabla \cdot\left(\boldsymbol{\sigma}_{0} \cdot \boldsymbol{u}\right)=2\left(\eta(\boldsymbol{r})-\eta_{0}\right) \boldsymbol{E}: \boldsymbol{E}_{0} .
$$


In both Stokes flow problems, i.e., those for constant viscosity and where viscosity varies, the particle shape is the same so that the fluid-filled domains $V$ are bounded by the surface of the particle $S_{p}$ and a bounding surface at large distances $S_{\infty}$. Then we integrate over the domain and utilize the Divergence Theorem, after which Eq. (14) becomes

$$
-\int_{S_{p}} \boldsymbol{n} \cdot \boldsymbol{\sigma} \cdot \boldsymbol{u}_{0} d S+\int_{S_{p}} \boldsymbol{n} \cdot \boldsymbol{\sigma}_{0} \cdot \boldsymbol{u} d S=2 \int_{V}\left[\eta(\boldsymbol{r})-\eta_{0}\right] \boldsymbol{E}: \boldsymbol{E}_{0} d V,
$$

where we recall that $\boldsymbol{n}$ is the unit vector directed into the fluid. In Eq. (15), integration over the bounding surface at infinity vanishes due to the decaying velocity and stress fields associated with Stokes flow problems (we assume the viscosity tends to a constant in the far field). Equation (15) is the form of the reciprocal theorem we utilize in the rest of the paper.

Now we apply Eq. (15) to translation and rotation of a particle, where the boundary condition on $S_{p}$ for the main problem is $\boldsymbol{u}=\boldsymbol{U}+\boldsymbol{\Omega} \wedge \mathbf{r}$ and for the constant viscosity problem is $\boldsymbol{u}_{0}=\boldsymbol{U}_{0}+\boldsymbol{\Omega}_{0} \wedge \mathbf{r}$. Then using the definitions (4) we have

$$
\boldsymbol{F} \cdot \boldsymbol{U}_{0}-\boldsymbol{F}_{0} \cdot \boldsymbol{U}+\boldsymbol{T} \cdot \boldsymbol{\Omega}_{0}-\boldsymbol{T}_{0} \cdot \boldsymbol{\Omega}=2 \int_{V}\left[\eta_{0}-\eta(\boldsymbol{r})\right] \boldsymbol{E}: \boldsymbol{E}_{0} d V
$$

where $\boldsymbol{F}$ and $\boldsymbol{T}$ and $\boldsymbol{F}_{0}$ and $\boldsymbol{T}_{0}$ are, respectively, hydrodynamic forces and torques on the particle for the variable viscosity and constant viscosity cases. Equation (16) is the starting point for the subsequent analysis of the influence of the viscosity field $\eta(\boldsymbol{r})$ on the force and torque on a particle.

Owing to the linearity of the Stokes equations and the boundary conditions, which involve both translation with velocity $\boldsymbol{U}$ (or $\boldsymbol{U}_{0}$ ) and rotation with angular velocity $\boldsymbol{\Omega}$ (or $\boldsymbol{\Omega}_{0}$ ), we can write the rate of strain tensor as a sum of two tensors, $\boldsymbol{E}^{U}$, due to translation, and $\boldsymbol{E}^{\Omega}$, due to rotation. We will also define corresponding third-order tensors, $\mathcal{E}^{\beta}$, which we shall refer to as the normalized strain rate tensors, such that

$$
\boldsymbol{E}=\boldsymbol{E}^{U}+\boldsymbol{E}^{\Omega}=\mathcal{E}^{U} \cdot \boldsymbol{U}+\mathcal{E}^{\Omega} \cdot \boldsymbol{\Omega} \quad \text { and } \quad \boldsymbol{E}_{0}=\boldsymbol{E}_{0}^{U}+\boldsymbol{E}_{0}^{\Omega}=\mathcal{E}_{0}^{U} \cdot \boldsymbol{U}_{0}+\mathcal{E}_{0}^{\Omega} \cdot \boldsymbol{\Omega}_{0} .
$$

Using the linearity of the problem, it follows from Eq. (16) that we can eliminate the otherwise arbitrary vectors $\boldsymbol{U}_{0}$ and $\boldsymbol{\Omega}_{0}$. Thus, we find independent equations for $\boldsymbol{F}$ and $\boldsymbol{T}$. Using Eq. (17), we write the resulting equations in matrix form:

$$
\begin{aligned}
\qquad\left(\begin{array}{l}
\boldsymbol{F} \\
\boldsymbol{T}
\end{array}\right) & =\boldsymbol{R}_{0} \cdot\left(\begin{array}{l}
\boldsymbol{U} \\
\boldsymbol{\Omega}
\end{array}\right)+2 \eta_{0} \int_{V}\left(1-\frac{\eta(\boldsymbol{r})}{\eta_{0}}\right) \Lambda \mathrm{d} V \cdot\left(\begin{array}{l}
\boldsymbol{U} \\
\boldsymbol{\Omega}
\end{array}\right) \\
\text { with } \quad \boldsymbol{R}_{0} & \equiv\left(\begin{array}{ll}
\boldsymbol{R}_{0}^{F U} & \boldsymbol{R}_{0}^{F \Omega} \\
\boldsymbol{R}_{0}^{T U} & \boldsymbol{R}_{0}^{T \Omega}
\end{array}\right) \text { and } \boldsymbol{\Lambda} \equiv\left(\begin{array}{ll}
\mathcal{E}_{0}^{U} \cdot \mathcal{E}^{U} & \mathcal{E}_{0}^{U} \cdot \mathcal{E}^{\Omega} \\
\mathcal{E}_{0}^{\Omega}: \mathcal{E}^{U} & \mathcal{E}_{0}^{\Omega}: \mathcal{E}^{\Omega}
\end{array}\right),
\end{aligned}
$$

where for the purposes of a compact notation in Eq. (18) we have defined the double inner product $:$ of two third-order tensors that appear here according to $\mathcal{E}_{0}: \mathcal{E}=\mathcal{E}_{0(k j i)} \mathcal{E}_{j k \ell} \mathbf{e}_{i} \mathbf{e}_{\ell}$.

In Eq. (18) the matrix $\boldsymbol{R}_{0}$ is the grand resistance tensor of a particle translating and rotating in a fluid of uniform viscosity. The second term in Eq. (18) gives the correction to the resistance due to variations in viscosity. Unlike the usual case of a particle in a homogeneous fluid, the second-order tensor $\boldsymbol{\Lambda}$ does not depend solely on the geometry of the particle [10], but instead also depends on the viscosity variation. More generally, we now observe that $\left(\begin{array}{c}F \\ T\end{array}\right)=\boldsymbol{R} \cdot\left(\begin{array}{l}U \\ \Omega\end{array}\right)$, where, accounting for viscosity variations, the resistance tensor is

$$
\boldsymbol{R}=\boldsymbol{R}_{0}+2 \eta_{0} \int_{V}\left[1-\frac{\eta(\boldsymbol{r})}{\eta_{0}}\right] \boldsymbol{\Lambda} d V .
$$

Features and results derived from Eq. (19) are the main consequence of this work. 
For a spherical particle of radius $a$, it is well known that $\boldsymbol{R}_{0}^{F U}=-6 \pi \eta_{0} a \boldsymbol{I}, \boldsymbol{R}_{0}^{T \Omega}=-8 \pi \eta_{0} a^{3} \boldsymbol{I}$, and $\boldsymbol{R}_{0}^{F \Omega}=\boldsymbol{R}_{0}^{T U}=\mathbf{0}$. We next consider how these results change when there is a variation of viscosity.

\section{Analysis for the motion of a sphere}

The flow, $\boldsymbol{u}_{0}(\boldsymbol{r})$, due to a sphere of radius $a$ translating $(t r)$ and rotating $(r)$ in a constant viscosity fluid in the low-Reynolds-number flow limit, is given by [10]

$$
\begin{aligned}
\boldsymbol{u}_{0}(\boldsymbol{r}) & =\boldsymbol{u}_{0}^{t r}(\boldsymbol{r})+\boldsymbol{u}_{0}^{r}(\boldsymbol{r}), \\
\text { with } \quad \boldsymbol{u}_{0}^{t r}(\boldsymbol{r}) & =\left[\frac{3 a}{4 r}\left(\boldsymbol{I}+\frac{\boldsymbol{r} \boldsymbol{r}}{r^{2}}\right)+\frac{a^{3}}{4 r^{3}}\left(\boldsymbol{I}-\frac{3 \boldsymbol{r} \boldsymbol{r}}{r^{2}}\right)\right] \cdot \boldsymbol{U}_{0} \\
\text { and } \quad \boldsymbol{u}_{0}^{r}(\boldsymbol{r}) & =\boldsymbol{\Omega}_{0} \wedge \boldsymbol{r}\left(\frac{a}{r}\right)^{3} .
\end{aligned}
$$

Substituting Eqs. (20) in the definition of the rate of strain tensor, $\boldsymbol{E}_{0}=\frac{1}{2}\left(\boldsymbol{\nabla} \boldsymbol{u}_{0}+\left(\boldsymbol{\nabla} \boldsymbol{u}_{0}\right)^{T}\right)$ and using Eq. (17), we can express the normalized strain rate tensors as

$$
\begin{aligned}
& \mathcal{E}_{0(i j k)}^{U}=\frac{3 a r_{k}}{4 r^{3}}\left(\delta_{i j}-\frac{3 r_{i} r_{j}}{r^{2}}\right)+\frac{3 a^{3}}{4 r^{5}}\left[r_{k}\left(-\delta_{i j}+\frac{5 r_{i} r_{j}}{r^{2}}\right)-r_{i} \delta_{j k}-r_{j} \delta_{i k}\right] \\
& \mathcal{E}_{0(i j k)}^{\Omega}=-\frac{3 a^{3}}{2 r^{5}}\left(r_{i} r_{\ell} \epsilon_{\ell j k}+r_{j} r_{\ell} \epsilon_{\ell i k}\right) .
\end{aligned}
$$

With $\mathcal{E}_{0}$ at hand, we are almost ready to calculate the correction to the resistance tensor using Eq. (19). The two missing components are the viscosity variation, $\eta(\boldsymbol{r})$, and the normalized strain rate tensor, $\mathcal{E}$. We tend to these in the next section.

\section{PERTURBATION ANALYSIS}

In this section, a perturbation analysis is used to solve Eq. (1) or (3) for systems with small temperature and viscosity variations. We first consider the temperature distribution, which establishes the viscosity field about the particle. Then we consider the corresponding velocity distribution and evaluate the force and torque using the reciprocal theorem results from the preceding section.

Note that in the analysis below, since the Péclet number, Pe, is assumed small, we consider only the conductively dominated thermal field. In fact, it is well known that the detailed description of the temperature distribution in the low-Péclet-number limit requires singular perturbation techniques [16], since in the far-field convective effects are no longer negligible compared to conductive effects. We briefly discuss these details in Sec. IIIE and show that they introduce only corrections that are much smaller than the dominant terms calculated here. In particular, in the worst case they are $O\left(\mathrm{Pe}^{2}\right)$ smaller than the corrections calculated below. Thus, when presenting the calculation below, we treat the necessary expansions as regular perturbations, but recognize that a more detailed analysis is needed if higher-order terms are desired.

\section{A. The temperature field}

At steady state, in the reference frame of a hot object, due to the assumed small Péclet number, the thermal distortions induced by the flow are negligible and the temperature distribution is obtained by solving $k_{0} \nabla^{2} T=-q(\boldsymbol{r})$, where $q$ is the heat flux distributed on or inside the sphere surface, and $k_{0}$ is the thermal conductivity, which is assumed constant. We assume that, relative to axes fixed to the particle, the thermal distribution has a given structure, which is consistent with the thermal conductivity of the particle being much larger than the conductivity of the surrounding fluid. As a result, in spherical coordinates $(r, \theta, \phi)$, the temperature field can be written in terms of spherical 
harmonics (e.g., Ref. [17])

$$
T(\boldsymbol{r})=T_{0}+\sum_{\ell=0}^{\infty} \sum_{m=-\ell}^{m=\ell} \frac{q_{\ell m}}{k_{0}(2 \ell+1)}\left(\frac{1}{r}\right)^{\ell+1} Y_{\ell}^{m}(\theta, \phi),
$$

where $\theta$ is defined as the angle relative to a given orientation associated with temperature distribution of the particle, $\phi$ is the corresponding azimuthal angle, the spherical harmonics $Y_{\ell}^{m}(\theta, \phi) \propto$ $P_{\ell}^{m}(\cos \theta) e^{i m \phi}$, and $P_{\ell}^{m}$ are the associated Legendre polynomials. The complex coefficients $q_{\ell m}$ are the multipole moments of the internal heat flux distribution, which are given by integration of the heat flux distribution over the volume of the particle, $q_{\ell m}=\int Y_{\ell m}^{*}(\theta, \phi) r^{\ell} q(\boldsymbol{r}) d V$.

A combination of these coefficients can be related to physical properties such as a heat monopole, heat dipole, heat quadrupole, etc. In particular, $Q$ is the total heat flow from the particle, $Q=$ $\sqrt{4 \pi} q_{00}=\int q(\boldsymbol{r}) d V$. Also, the vector $\boldsymbol{\Delta}=\int \boldsymbol{r} q(\boldsymbol{r}) d V$ is a source dipole, and the second-order tensor $\boldsymbol{Q}=\int\left(3 \boldsymbol{r} \boldsymbol{r}-\boldsymbol{r}^{2} \boldsymbol{I}\right) q(\boldsymbol{r}) d V$ is a source quadrupole, which, by definition, is traceless.

Alternatively, to highlight the thermal characteristics of a "hot particle," the first few terms of a multipole expansion can be written compactly in terms of the characteristic change in temperature that defines the thermal disturbance caused by the particle, i.e., $\Delta T_{m}=Q /\left(4 \pi k_{0} a\right), \Delta T_{d}$ and $\Delta T_{q}$, respectively, for the monopole, dipole, and quadruple. Hence, using $\sim$ to denote a normalized dipole $\tilde{\Delta}$ and quadrupole $\tilde{Q}$, we can write

$$
T(\boldsymbol{r})-T_{0}=\Delta T_{m} \frac{a}{r}+\Delta T_{d} \frac{a^{2} \tilde{\boldsymbol{\Delta}} \cdot \boldsymbol{r}}{r^{3}}+\Delta T_{q} \frac{a^{3} \tilde{\boldsymbol{Q}}:\left(3 \boldsymbol{r} \boldsymbol{r}-r^{2} \boldsymbol{I}\right)}{6 r^{5}}+\cdots,
$$

where an analysis of the multipole expansion shows that

$$
Q=4 \pi k_{0} a \Delta T_{m}, \quad \Delta=4 \pi k_{0} a^{2} \Delta T_{d} \tilde{\Delta}, \quad \text { and } \quad \boldsymbol{Q}=4 \pi k_{0} a^{3} \Delta T_{q} \tilde{\boldsymbol{Q}} .
$$

Below we will show that for a sphere all moments higher than a quadrupole do not contribute to the leading-order corrections to the force and torque.

\section{B. The viscosity field}

The thermal field $T(\boldsymbol{r})$ can be applied to approximate the viscosity variation around a particle. To be concrete we denote the temperature far from the particle as $T_{0}$ and the viscosity far from the particle as $\eta_{0}$ (consistent with the reference viscosity introduced in earlier sections). For a heated particle it is then natural to take the typical change in temperature as $\Delta T_{m}$; we note that if $Q=\Delta T_{m}=0$, then it is natural to discard the monopole in the equations below and take the characteristic change in temperature as $\Delta T_{d}$ or $\Delta T_{q}$. For small temperature variations the viscosity can be expanded as a Taylor series around $T_{0}$ :

$$
\eta \approx \eta_{0}\left(1+\left.\frac{\Delta T_{m}}{\eta_{0}} \frac{\partial \eta}{\partial T}\right|_{T_{0}} \mathcal{T}(\boldsymbol{r})+\cdots\right) \quad \text { where } \quad \mathcal{T}(\boldsymbol{r})=\frac{T(\boldsymbol{r})-T_{0}}{\Delta T_{m}} .
$$

Introducing a small parameter $\epsilon$ that captures the (small) temperature and viscosity variations produced by a heated particle, we define

$$
\epsilon=-\left.\frac{\Delta T_{m}}{\eta_{0}} \frac{\partial \eta}{\partial T}\right|_{T_{0}}
$$

since typically $\frac{\partial \eta}{\partial T}<0$. Clearly, $\epsilon$ represents the fractional change of viscosity, $\frac{\Delta \eta}{\eta_{0}}$. With respect to $\epsilon$ the viscosity from Eq. (25) is written as

$\eta(\boldsymbol{r}) \approx \eta_{0}\left[1-\epsilon \mathcal{T}(\boldsymbol{r})+O\left(\epsilon^{2}\right)\right] \quad$ where $\quad \mathcal{T}(\boldsymbol{r})=\sum_{\ell=0}^{\infty} \sum_{m=-\ell}^{\ell} \frac{q_{\ell m}}{\Delta T_{m} k_{0}(2 \ell+1)}\left(\frac{1}{r}\right)^{\ell+1} Y_{\ell}^{m}(\theta, \phi)$. 
Alternatively, explicitly recognizing the first three thermal moments, then with respect to $\epsilon$ the viscosity variation from Eq. (25) is written

$\eta(\boldsymbol{r}) \approx \eta_{0}\left[1-\epsilon \mathcal{T}(\boldsymbol{r})+O\left(\epsilon^{2}\right)\right] \quad$ where $\quad \mathcal{T}(\boldsymbol{r})=\frac{a}{r}+\frac{\Delta T_{d}}{\Delta T_{m}} \frac{a^{2} \tilde{\boldsymbol{\Delta}} \cdot \boldsymbol{r}}{r^{3}}+\frac{\Delta T_{q}}{\Delta T_{m}} \frac{a^{3} \tilde{\boldsymbol{Q}}:\left(3 \boldsymbol{r} \boldsymbol{r}-r^{2} \boldsymbol{I}\right)}{6 r^{5}}$

\section{A perturbation expansion for the velocity distribution}

For the velocity and the pressure fields, which now depend on the viscosity distribution $\eta(\boldsymbol{r})$, we can write regular perturbation expansions:

$\boldsymbol{u}(\boldsymbol{r} ; \eta(\boldsymbol{r}))=\boldsymbol{u}_{0}(\boldsymbol{r})+\epsilon \boldsymbol{u}_{1}(\boldsymbol{r})+\epsilon^{2} \boldsymbol{u}_{2}(\boldsymbol{r})+\cdots \quad$ and $\quad p(\boldsymbol{r} ; \eta(\boldsymbol{r}))=p_{0}(\boldsymbol{r})+\epsilon p_{1}(\boldsymbol{r})+\epsilon^{2} p_{2}(\boldsymbol{r})+\cdots$.

The zeroth-order solution is that for the well-known homogeneous Stokes equation with a constant viscosity. The variation of viscosity is captured asymptotically by $\epsilon$, with the caveat noted below in Sec. III $E$ in the discussion of the temperature field that properly accounting for the far field requires singular perturbation techniques; since the far field does not contribute to the integrals below, we neglect these complications here.

The expansions for the rate-of-strain tensor and the corresponding expansion for the normalized strain rate tensor are the following (the possible superscripts $U$ and $\Omega$ on $\mathcal{E}$ have been suppressed)

$$
\boldsymbol{E}=\boldsymbol{E}_{0}+\epsilon \boldsymbol{E}_{1}+\epsilon^{2} \boldsymbol{E}_{2}+\cdots \quad \text { and } \quad \mathcal{E}=\mathcal{E}_{0}+\epsilon \mathcal{E}_{1}+\epsilon^{2} \mathcal{E}_{2}+\cdots
$$

Thus, using Eq. (18) we have

$$
\begin{aligned}
& \qquad\left(\begin{array}{l}
\boldsymbol{F} \\
\boldsymbol{T}
\end{array}\right)=\left(\boldsymbol{R}_{0}+\epsilon \boldsymbol{R}_{1}+O\left(\epsilon^{2}\right)\right) \cdot\left(\begin{array}{l}
\boldsymbol{U} \\
\boldsymbol{\Omega}
\end{array}\right) \\
& \text { with } \quad \boldsymbol{R}_{1} \equiv 2 \eta_{0} \int \mathcal{T}(\boldsymbol{r}) \boldsymbol{\Lambda}_{0} d V=2 \eta_{0} \int \mathcal{T}(\boldsymbol{r})\left(\begin{array}{ll}
\mathcal{E}_{0}^{U}: \mathcal{E}_{0}^{U} & \mathcal{E}_{0}^{U} \cdot \mathcal{E}_{0}^{\Omega} \\
\mathcal{E}_{0}^{\Omega}: \mathcal{E}_{0}^{U} & \mathcal{E}_{0}^{\Omega}: \mathcal{E}_{0}^{\Omega}
\end{array}\right) d V,
\end{aligned}
$$

where $\boldsymbol{R}_{1}$ can be evaluated in terms of the thermal field and known quantities for the constant viscosity zeroth-order solution. This equation is our main perturbation result since, as discussed below, it introduces the first effects of viscosity variations on the dynamics of an arbitrarily shaped hot particle.

\section{Symmetries and expected properties of the resistance tensors}

A few remarks are in order about the corrections introduced by viscosity variations in the neighborhood of a particle. First, as Eq. (31b) makes clear, the second-order tensor contributions $\boldsymbol{R}_{1}^{F U}$ and $\boldsymbol{R}_{1}^{T \Omega}$ are symmetric. Second, we can observe that $\mathcal{E}_{0}^{U} \cdot \mathcal{E}_{0}^{\Omega}=\left(\mathcal{E}_{0}^{\Omega}: \mathcal{E}_{0}^{U}\right)^{T}$ so that the tensor $\boldsymbol{R}_{1}^{F \Omega}$ is the transpose of $\boldsymbol{R}_{1}^{T U}$. Of course, these properties have been shown in Sec. II B 2 to be true for an arbitrarily shaped particle and a given viscosity distribution, not simply for a small change in viscosity.

In addition, for the case of a spherical particle, several additional conclusions can be drawn. For example, the tensor $\boldsymbol{R}_{1}^{F \Omega}$ involves rotation and the tensor $\boldsymbol{R}_{1}^{T U}$ involves torque. As a consequence, both $\boldsymbol{R}_{1}^{F \Omega}$ and $\boldsymbol{R}_{1}^{T U}$ are pseudotensors (their features depend on the choice of the right-hand rule). The latter fact allows us to conclude that $\boldsymbol{R}_{1}^{F \Omega}$ and $\boldsymbol{R}_{1}^{T U}$ can involve only a contribution from a thermal dipole (and the permutation tensor) as we calculate explicitly below. Similarly, $\boldsymbol{R}_{1}^{F U}$ and $\boldsymbol{R}_{1}^{T \Omega}$ must be independent of the thermal dipole but can depend on the monopole and quadrupole terms. 


\section{E. Remarks about the low Péclet number assumption}

A few comments are needed to justify the use of the low Péclet number description used to represent the thermal field around a translating hot particle. To be specific we consider a spherical particle of radius $a$ translating at speed $U$ and define the Péclet number as $\mathrm{Pe}=U a / \kappa$, where $\kappa$ is the thermal diffusion coefficient. We are assuming $P e \ll 1$.

Because the temperature distribution requires the solution of a convective-diffusion equation, then when $\mathrm{Pe} \ll 1$, the temperature field near the particles is conductively dominated, i.e., $\nabla^{2} T=0$. However, sufficiently far from the particle, where $T \rightarrow T_{0}$, the description of the variations of the temperature field requires inclusion of convective effects [16]. This far field occurs at a length scale $O(a / \mathrm{Pe})$. A detailed analysis requires singular perturbation methods though order-of-magnitude estimates are sufficient to show that the convective contributions do not affect our conclusions. For example, Eq. (31b) is an exact integral statement whose evaluation requires the temperature distribution. Let us consider the monopole thermal field, which breaks down at a length scale $O(a / \mathrm{Pe})$. The other contributions to the kernel vary at most as $r^{-4}$ as $r \rightarrow \infty$ (these are the contributions from $\left.\Lambda^{U U}\right)$. The corresponding contributions in the far field to the resistance tensor $\boldsymbol{R}_{1}$ have magnitude $O\left(r^{-1} \cdot r^{-4} \cdot r^{3}\right)=O\left(\mathrm{Pe}^{2}\right)$. Such corrections can be neglected in the limit $\mathrm{Pe} \ll 1$.

\section{CORRECTIONS TO THE GRAND RESISTANCE TENSOR FOR A SPHERICAL PARTICLE}

We now evaluate the change in the force and torque on a sphere produced by a change in the surrounding viscosity. We use the velocity and corresponding strain rate fields determined, respectively, in Eqs. (20) and (21). Therefore, we calculate the various terms in $\boldsymbol{R}_{1}$ of Eq. (31). First, we find

$$
\begin{aligned}
& \left(\mathcal{E}_{0}^{U} \cdot \mathcal{E}_{0}^{U}\right)_{i j}=\frac{9 a^{6}}{8 r^{8}}\left\{\left[3\left(\frac{r}{a}\right)^{4}-6\left(\frac{r}{a}\right)^{2}+2\right] \frac{r_{i} r_{j}}{r^{2}}+\delta_{i j}\right\} \\
& \left(\mathcal{E}_{0}^{U}: \mathcal{E}_{0}^{\Omega}\right)_{i j}=\left(\mathcal{E}_{0}^{\Omega} \bullet \mathcal{E}_{0}^{U}\right)_{j i}=\frac{9 a^{6}}{4 r^{8}} \epsilon_{i j k} r_{k} \\
& \left(\mathcal{E}_{0}^{\Omega} \cdot \mathcal{E}_{0}^{\Omega}\right)_{i j}=\frac{9 a^{6}}{2 r^{6}}\left(\delta_{i j}-\frac{r_{i} r_{j}}{r^{2}}\right) .
\end{aligned}
$$

The different components of Eq. (32) can be written in terms of spherical harmonics, which involve only terms corresponding to the zeroth-, first-, and second-order Legendre polynomials. Thus, from the orthogonality of the spherical harmonics we need to consider only the monopole, dipole and quadrupole parts of the thermal distribution [Eq. (23)], and higher-order moments do not contribute. Substituting these results along with the viscosity expansion of Eq. (28) in Eq. (31), we find

$$
\begin{aligned}
& \boldsymbol{R}_{1}^{F U}=\frac{5 \pi \eta_{0} a}{2} \boldsymbol{I} \\
& \boldsymbol{R}_{1}^{F \Omega}=-\boldsymbol{R}_{1}^{T U}=\pi \eta_{0} a^{2} \frac{\Delta T_{d}}{\Delta T_{m}} \boldsymbol{\epsilon} \cdot \tilde{\Delta} \\
& \boldsymbol{R}_{1}^{T \Omega}=6 \pi \eta_{0} a^{3}\left(\boldsymbol{I}-\frac{1}{15} \frac{\Delta T_{q}}{\Delta T_{m}} \tilde{\boldsymbol{Q}}\right),
\end{aligned}
$$

where $\boldsymbol{\epsilon}=\epsilon_{i j k} \boldsymbol{e}_{i} \boldsymbol{e}_{j} \boldsymbol{e}_{k}$ is the third-order permutation tensor. In Eq. (33) we have written the various multipole moments, in terms of the more physical temperature ratios that characterize the heat distribution on the particle. Although it is possible for a quadrupolar contribution to appear in Eq. (33a), it turns out the term integrates to zero. 
(a)

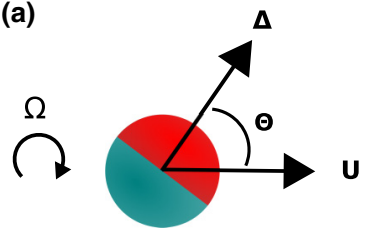

(b)

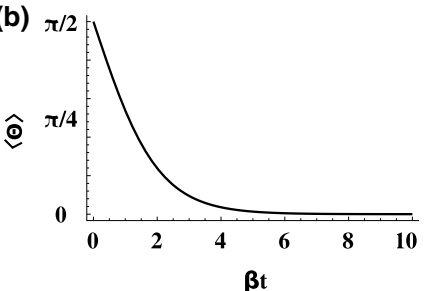

FIG. 1. (a) Schematic representation of a Janus particle. The angle between the direction of the temperature variation, $\boldsymbol{\Delta}$, and the velocity, $\boldsymbol{U}$, is denoted $\Theta$. (b) The average angle, $\langle\Theta\rangle$, as a function of normalized time, $\beta t$, where $\beta$ is the relaxation time defined in Eq. (36) and the average, presented in Eq. (38), is taken over all possible initial angles.

Equation (33) is true for a spherical particle that has a temperature distribution and a small relative change in viscosity. We note that the translation correction tensor, $\boldsymbol{R}_{1}^{F U}$, is symmetric and has a contribution only from a heat monopole, whereas the rotational tensor, $\boldsymbol{R}_{1}^{T \Omega}$, is symmetric and includes an additional quadrupole contribution. Only $\boldsymbol{R}_{1}^{F \Omega}$ and $\boldsymbol{R}_{1}^{T U}$ are influenced by the thermal dipole. These features are consistent with the mathematical properties indicated in Sec. III D. We will now look at a few specific examples of particles with various thermal distributions.

\section{A. Uniformly hot particle}

For a uniformly hot particle, the viscosity distribution is isotropic, and all multipoles except the monopole, $\Delta T_{m}$ (or $Q$ ), are zero. The force and the torque are

$$
\begin{aligned}
& \boldsymbol{F} \simeq-6 \pi \eta_{0} a\left(1-\frac{5 \epsilon}{12}\right) \boldsymbol{U} \\
& \boldsymbol{T} \simeq-8 \pi \eta_{0} a^{3}\left(1-\frac{3 \epsilon}{4}\right) \boldsymbol{\Omega} .
\end{aligned}
$$

As expected on physical grounds, for a spherical particle in a radially symmetric viscosity field, there is no coupling between translation and rotation. Also, the magnitude of the force and torque are smaller than the constant viscosity case as lower viscosity fluid is adjacent to a hot particle.

\section{B. Hot Janus particle}

In a Janus particle half of the sphere is hot and the other half has the same temperature as the ambient fluid. For such a case there will be a nonzero thermal monopole (e.g., $\Delta T_{m}$ or $Q$ ), and a thermal dipole, $\tilde{\boldsymbol{\Delta}}$ with $\Delta T_{d} \neq 0$; see Fig. 1(a). There will be no quadrupole, $\tilde{\boldsymbol{Q}}=\mathbf{0}$. The correction to the force, and the torque will be the same as in Eq. (34). There will, however, be an additional correction due to coupling between rotation and translation, such that

$$
\begin{aligned}
& \boldsymbol{F} \simeq-6 \pi \eta_{0} a\left(1-\frac{5 \epsilon}{12}\right) \boldsymbol{U}-\epsilon \pi \eta_{0} a^{2} \frac{\Delta T_{d}}{\Delta T_{m}} \tilde{\boldsymbol{\Delta}} \wedge \boldsymbol{\Omega}, \\
& \boldsymbol{T} \simeq-8 \pi \eta_{0} a^{3}\left(1-\frac{3 \epsilon}{4}\right) \boldsymbol{\Omega}+\epsilon \pi \eta_{0} a^{2} \frac{\Delta T_{d}}{\Delta T_{m}} \tilde{\boldsymbol{\Delta}} \wedge \boldsymbol{U} .
\end{aligned}
$$

Such coupling of the translational and rotational motions for spherical particles when there are local viscosity variations is absent in an isothermal fluid. Similar coupling terms have been derived in the case of a slip-stick particle [18]. In both cases the origin of the coupling comes from the breaking of spherical symmetry around the particle. 
Recall that the thermal dipole indicates the "axis" of the viscosity variation, and as the Péclet number is small, the viscosity variations follow the rotation of the particle. We can observe from Eq. (35) that for a torque-free spherical particle there is no coupling of translation and rotation if the thermal dipole is oriented in the direction of translation. Furthermore, for a given external force and zero external torque, the orientation of the thermal dipole will change over time until it is oriented in the direction of the force. Therefore, the viscosity variation acts as a restoring torque to align $\tilde{\Delta}$ and $\boldsymbol{U}$. In cases where the Janus particle is small enough to be affected by thermal forces, this restoring torque would hinder rotational diffusion. Physically, the alignment of the thermal dipole with the translation direction is consistent with the translation to occur in the direction of the lower viscosity fluid, which is in the direction indicated by $\tilde{\boldsymbol{\Delta}}$.

We can determine the time-dependent response for a particle translating with velocity $\boldsymbol{U}$ by setting $\boldsymbol{T}=\mathbf{0}$ in Eq. (35b). If $\Theta(t)$ is the angle between the velocity and the dipole $(0 \leqslant \Theta \leqslant \pi)$, then we find

$$
\dot{\Theta}=-\beta \sin \Theta \quad \text { with } \quad \beta \equiv \frac{\epsilon U}{8 a\left(1-\frac{3 \epsilon}{4}\right)} \frac{\Delta T_{d}}{\Delta T_{m}} \simeq \frac{\epsilon U}{8 a} \frac{\Delta T_{d}}{\Delta T_{m}}
$$

With $\Theta(0)=\Theta_{0}$, the initial angle between the dipole and the velocity vector, the solution to this equation is

$$
\Theta(t)=2 \operatorname{arccot}\left[\cot \left(\Theta_{0} / 2\right) \mathrm{e}^{\beta t}\right] \stackrel{t \gg 1 / \beta}{\longrightarrow} \frac{2 \mathrm{e}^{-\beta t}}{\cot \left(\Theta_{0} / 2\right)} .
$$

At long times the angle decays exponentially to zero such that the dipole will be oriented with the velocity vector, and according to Eq. (35) there will be no further coupling between rotation and translation. We can see this result clearly after averaging Eq. (37) over all initial angles, $\Theta_{0}$ :

$$
\langle\Theta(t)\rangle=\frac{\pi}{1+\mathrm{e}^{\beta t}} .
$$

This result is plotted in Fig. 1(b).

\section{Quadrupole distribution}

As is clear from Eq. (33) for the motion of a hot sphere, all multipole terms that are higher order than a quadrupole will not contribute to the resistance tensor. To simplify matters, let us now look at a heat distribution for which there is no dipolar term, $\tilde{\Delta}=\mathbf{0}$. We note that in cases where a dipole is present, it will not change the results below, but will merely add a coupling term between the torque and the force, as is shown above in Eq. (35). In the absence of such dipole-induced coupling of translation and rotation, a torque-free sphere will translate but not rotate, independent of the details of the quadrupolar temperature distribution.

Two examples of quadrupolar distributions are sketched in Fig. 2. We will examine them in order to give intuition to the general form of the solution. The correction to the force depends only on the monopole term and is given by Eq. (34). Since the dipolar term is absent, rotation and translation are uncoupled. The correction to the torque depends on the quadrupole orientation and magnitude. For a particle with an axial quadrupolar distribution oriented along the $z$ direction, as shown in Fig. 2(a), the correction to the torque is

$$
\boldsymbol{T} \simeq-8 \pi \eta_{0} a^{3}\left[\left(1-\frac{3 \epsilon}{4}\right) \boldsymbol{I}+\frac{\epsilon}{20} \frac{\Delta T_{q}}{\Delta T_{m}}\left(\begin{array}{rrr}
-\frac{1}{2} & 0 & 0 \\
0 & -\frac{1}{2} & 0 \\
0 & 0 & 1
\end{array}\right)\right] \cdot \boldsymbol{\Omega} .
$$




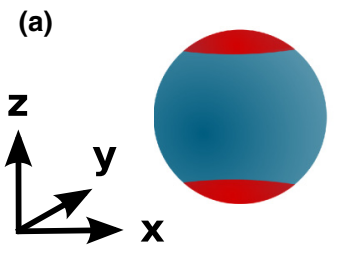

(b)

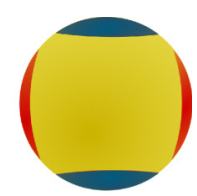

FIG. 2. Two examples of quadrupolar temperature distributions for spherical particles. Neither example possesses a dipole term. In both cases there will be an additional monopole terms corresponding to the total heat flow according to the monopole temperature difference $\Delta T_{m}$, where $Q /\left(4 \pi k_{0} a\right)=\Delta T_{m}$. (a) Two hot spherical caps (red), one each at the north and south poles of the sphere, which is shown aligned in the sketch along the $z$ direction. (b) The entire particle is at a higher temperature (yellow) than the ambient fluid except for two caps at the poles aligned in the $z$ direction; in addition two "circular" caps at an even higher temperature (red) are aligned in the $x y$ plane in the sketch. In both examples we characterize the quadrupolar temperature distribution by $\Delta T_{q}$.

For a particle with a quadrupole laying in the $x z$ plane, as is depicted in Fig. 2(b), the correction to the torque is given by

$$
\boldsymbol{T} \simeq-8 \pi \eta_{0} a^{3}\left[\left(1-\frac{3 \epsilon}{4}\right) \boldsymbol{I}+\frac{\epsilon}{20} \frac{\Delta T_{q}}{\Delta T_{m}}\left(\begin{array}{lll}
0 & 0 & 1 \\
0 & 0 & 0 \\
1 & 0 & 0
\end{array}\right)\right] \cdot \boldsymbol{\Omega} .
$$

By construction, $Q$ is symmetric and traceless, hence the most general result for the quadrupole tensor will have five independent components. Any additional temperature variation on the sphere will not alter the results above as long as it is a higher multipole.

\section{DISCUSSION}

We have presented a method to derive analytic corrections to the force and torque acting on a particle that produces temperature gradients, and corresponding viscosity gradients, in a fluid when both the Reynolds number and Péclet number are small. The latter assumption eliminates any history dependence in the viscosity distribution and ties the viscosity field to the (quasisteady) temperature field of the particle. First, the results are presented for particles with arbitrary shape. For small temperature variations, we developed the solution by applying a perturbation method in terms of a small parameter $\epsilon$ representing a small change in viscosity. The first term in the perturbation expansion is the well-known constant viscosity case, while we also determine the $O(\epsilon)$ terms for the hydrodynamic force and torque. Second, detailed results were then given for spherical particles with different temperature distributions.

Our approach utilizes the reciprocal theorem, which avoids complexities due to solving for the flow field. The $O(\epsilon)$ corrections we obtain only require knowledge of the $O(1)$ fields. In particular, the first corrections to the hydrodynamic force and torque on a spherical particle with uniform and nonuniform temperatures are evaluated; the thermal characterizations require only the first three singularities, as described by a thermal monopole, dipole, and quadrupole. Among other results for spherical particles, we find for dipolar thermal fields that there is coupling of the translational and rotational motions when there are local viscosity variations; such coupling is absent in an isothermal fluid. Also, we can contrast our result for the force-translation resistance tensor, which finds a correction $-\frac{5}{12} \epsilon$, with the spherical shell model of Chakraborty et al. [6] who, in our notation, obtained $-\frac{193}{486} \epsilon$; the approximate results gives a value 0.95 of our asymptotic value. Finally, although we have focused on the hydrodynamics of a hot particle, our general analysis can be applied to the problems where a particle moves in a material with inhomogeneous rheological properties; e.g., 


\section{MOTION OF A HOT PARTICLE IN VISCOUS FLUIDS}

thermophoresis of rigid particles is one problem of this type. We hope to develop further results in this spirit in future work.

\section{ACKNOWLEDGMENTS}

We thank F. Cichos, U. Khadka, M. Selmke, and H. Yang for introducing us to this question and H. Diamant, T. Goldfriend, and H. Moyses for helpful feedback.

[1] R. Golestanian, T. B. Liverpool, and A. Ajdari, Designing phoretic micro-and nano-swimmers, New J. Phys. 9, 126 (2007).

[2] S. Iacopini and R. Piazza, Thermophoresis in protein solutions, Europhys. Lett. 63, 247 (2003).

[3] R. Piazza and A. Parola, Thermophoresis in colloidal suspensions, J. Phys.: Condens. Matter 20, 153102 (2008).

[4] A. Würger, Thermophoresis in Colloidal Suspensions Driven by Marangoni Forces, Phys. Rev. Lett. 98, 138301 (2007).

[5] T. Bickel, A. Majee, and A. Würger, Flow pattern in the vicinity of self-propelling hot Janus particles, Phys. Rev. E 88, 012301 (2013).

[6] D. Chakraborty, M. V. Gnann, D. Rings, J. Glaser, F. Otto, F. Cichos, and K. Kroy, Generalised Einstein relation for hot Brownian motion, Europhys. Lett. 96, 60009 (2011).

[7] D. Rings, R. Schachoff, M. Selmke, F. Cichos, and K. Kroy, Hot Brownian Motion, Phys. Rev. Lett. 105, 090604 (2010).

[8] R. T. Schermer, C. C. Olson, J. P. Coleman, and F. Bucholtz, Laser-induced thermophoresis of individual particles in a viscous liquid, Opt. Express 19, 10571 (2011).

[9] A. Ansari and S. Morris, The effects of a strongly temperature-dependent viscosity on Stokes's drag law: experiments and theory, J. Fluid Mech. 159, 459 (1985).

[10] J. Happel and H. Brenner, Low Reynolds Number Hydrodynamics (Prentice-Hall, Englewood Cliffs, NJ, 1965), p. 85.

[11] B. P. Ho and L. G. Leal, Migration of rigid spheres in a two-dimensional unidirectional shear flow of a second-order fluid, J. Fluid Mech. 76, 783 (1976).

[12] L. G. Leal, Particle motions in a viscous fluid, Annu. Rev. Fluid Mech. 12, 435 (1980).

[13] L. E. Becker, G. H. McKinley, and H. A. Stone, Sedimentation of a sphere near a plane wall: weak non-Newtonian and inertial effects, J. Non-Newtonian Fluid Mech. 63, 201 (1996).

[14] P. M. Lovalenti and J. F. Brady, The hydrodynamic force on a rigid particle undergoing arbitrary timedependent motion at small Reynolds number, J. Fluid Mech. 256, 561 (1993).

[15] M. Teubner, The motion of charged colloidal particles in electric fields, J. Chem. Phys. 76, 5564 (1982).

[16] L. G. Leal, Advanced Transport Phenomena: Fluid Mechanics and Convective Transport Processes (Cambridge University Press, Cambridge, 2007).

[17] J. D. Jackson, Classical Electrodynamics (John Wiley, New York, 1999).

[18] J. W. Swan and A. S. Khair, On the hydrodynamics of 'slip-stick' spheres, J. Fluid Mech. 606, 115 (2008). 